\title{
Convergence internationale et diversification interne des modèles scolaires
}

International convergence and internal diversification in educational models Convergencia internacional y diversificación interna de los modelos escolares

\section{Bernard Charlot}

\section{OpenEdition}

\section{Journals}

Édition électronique

URL : https://journals.openedition.org/ries/785

DOI : $10.4000 /$ ries.785

ISSN : 2261-4265

Éditeur

France Education international

Édition imprimée

Date de publication : 1 décembre 2009

Pagination : 123-127

ISBN : 978-2-8542-0577-0

ISSN : $1254-4590$

\section{Référence électronique}

Bernard Charlot, "Convergence internationale et diversification interne des modèles scolaires », Revue internationale d'éducation de Sèvres [En ligne], 52 I décembre 2009, mis en ligne le 01 décembre 2012, consulté le 27 avril 2022. URL : http://journals.openedition.org/ries/785 ; DOI : https://doi.org/ $10.4000 /$ ries.785

(c) Tous droits réservés 


\title{
Convergence internationale et diversification interne des modèles scolaires
}

\section{Bernard Charlot}

\author{
Pendant la seconde moitié du $\mathrm{XX}^{\mathrm{e}}$ siècle, l'école, \\ initialement présentée comme la « solution », est progressivement \\ devenue un problème de grande dimension. \\ Rui Canário ${ }^{1}$
}

En mars 2009, la Revue internationale d'éducation de Sèvres éditée par le Centre international d'études pédagogiques (CIEP) a organisé des rencontres internationales sur le thème "Un seul monde, une seule école ? ». La réponse apportée à cette question par les cent trente experts venus de trente pays a été consensuelle et est confirmée par la lecture des communications, disponibles sur Internet ${ }^{2}$. Le numéro 50 de la revue, daté d'avril 2009, consacré aux pratiques pédagogiques et valeurs culturelles, aboutissait d'ailleurs aux mêmes conclusions ${ }^{3}$.

\section{DEUX RÉPONSES : PLURALITÉ ET CONVERGENCE DES MODĖLES}

Premier moment de la réponse : non, il n'y a pas une seule école, pas plus qu'il n'y a un seul monde. La diversité des écoles apparaît dès qu'on s'engage dans la description, à quelque niveau que ce soit. Diverse est l'ampleur des problèmes à résoudre : scolariser environ 210 millions d'élèves en Chine et 950000 en Finlande. Diverses, également, sont les pratiques institutionnelles et pédagogiques : au Danemark, les élèves ne reçoivent pas de notes individuelles avant l'âge de 14 ans ; au Brésil, certaines écoles maternelles privées organisent déjà de petites épreuves préparant au vestibular, examen d'entrée à l'université.

\footnotetext{
1. Intervention lors du colloque « Un seul monde, une seule école ? Les modèles scolaires à l'épreuve de la mondialisation » 5-7 mars 2009.

2. Voir : http://www.ciep.fr/ries/colloque-2009/contributions.php

3. Cet article prend appui, essentiellement, sur les contributions au colloque, ainsi que sur le numéro 50 de la Revue Internationale d'Éducation de Sévres « Pratiques pédagogiques et valeurs culturelles ». Nous sommes redevable à tous les auteurs. Pour ne pas alourdir le texte et la bibliographie, nous citerons seulement ceux à qui nous empruntons directement une information ou une idée, avec la référence « RIE 50 », lorsqu’il s'agit du numéro de la revue. Pour les autres auteurs, nous utiliserons le procédé habituel (nom et date), renvoyant à la bibliographie.
} 
Divers, enfin, est le vécu des élèves. Lorsqu'ils arrivent à l'école le matin, les petits Napolitains vont directement dans leur classe, seuls ou avec leurs parents, et y resteront toute la journée, y compris pour déjeuner et pour les moments de récréation (Longo et Roche, RIE 50). Les jeunes Français entrent dans l'école sans leurs parents, vont dans la cour et rejoindront leur classe tous ensemble, avec leur professeur ; ils retourneront dans la cour pour la récréation. Les élèves anglais commencent leur journée par l'assembly, réunion de tous les élèves de l'école, assis en tailleur et les bras croisés, écoutant des histoires édifiantes et assistant à la remise de certificats ou de médailles (Raveaud, 2006).

Toutefois, la réponse comporte un second moment : dans de nombreux pays, les écoles sont actuellement engagées dans des processus de transformation, hybridation, rupture. Elles tentent de résoudre des problèmes, de répondre à des exigences, d'affronter des défis, qui sont souvent les mêmes dans des pays aux modèles scolaires pourtant différents. Ces problèmes, exigences et défis sont des effets de la globalisation : l'interdépendance et la concurrence économiques croissantes entre les pays incitent chacun d'entre eux à tenter d'optimiser les performances de son école. Dans la mesure où toutes ces tentatives s'opèrent dans la logique de la globalisation, elles induisent des convergences entre les modèles scolaires. Ceux-ci se transforment, parfois se déforment, "à l'épreuve de la mondialisation ", pour reprendre les termes du sous-titre du colloque.

Cet effet de la globalisation est accentué par les évaluations internationales : avant tout PISA, avec les adolescents de quinze ans, mais aussi, aujourd'hui, PIRLS, avec les enfants de dix ans et la classification de Shanghaï, pour les universités. La hiérarchie internationale établie par ces évaluations, qui est censée être celle de la qualité des modèles scolaires, amène certains pays à adopter les standards internationaux utilisés par ces évaluations, voire à introduire dans leurs modèles les modalités organisationnelles et pédagogiques qui leur semblent être la source du succès des pays les mieux classés : aux convergences induites par la globalisation s'ajoutent des effets de transposition consciente et volontaire.

Les modèles scolaires sont pluriels, aujourd'hui encore, mais ils sont travaillés par des processus, implicites ou explicites, qui esquissent des convergences : telle est la conclusion de l'analyse descriptive. Peut-on avancer davantage et produire des éléments d'explication, au-delà de cette analyse descriptive ? C'est l'ambition de cet article.

\section{FONDEMENTS HISTORICO-CULTURELS DE LA DIVERSITÉ DES MODÈLES SCOLAIRES}

Pendant longtemps, la comparaison entre les systèmes scolaires s'est centrée sur les aspects organisationnels et a privilégié l'outil statistique. Cette approche était utile pour les administrateurs de l'éducation et les responsables 
politiques, mais elle a produit peu d'intelligibilité sur les modèles scolaires. L'approche actuelle tend à privilégier l'identification des valeurs culturelles qui imprègnent et fondent les modèles scolaires. On considère aujourd'hui que "les objectifs et la structure des systèmes éducatifs découlent de valeurs sociales et politiques profondément enracinées dans une société » (MacNess, RIE 50). La diversité des modèles serait la conséquence de la diversité de ces valeurs.

L'analyse comparative des modèles anglais et français est sans doute aujourd'hui la plus avancée, grâce aux travaux de chercheurs des deux pays, notamment P. Broadfoot, M. Osborn, R. Alexander, M. Raveaud et C. Planel. L'école française reflète les principes de la Nation et de la République : égalité, universalisme, citoyenneté, solidarité, alors que l'école anglaise est fondée sur des valeurs individuelles et communautaires. En France, on considère donc que tous les enfants doivent atteindre le même niveau et qu'il faut les traiter de la même façon. L'Angleterre pratique au contraire une pédagogie différenciée et individualisée en fonction des capacités et besoins de l'enfant. À cette première différence s'en ajoute une autre : en France prévaut la rationalité et un certain intellectualisme; en Angleterre, l'empirisme. Aussi l'enseignement français est-il très structuré, formel, fondé sur les cours, la transmission et le travail, alors que l'enseignement anglais laisse une large place à l'apprentissage par le jeu et la découverte et tend à négliger la formalisation des concepts. Ces valeurs et principes induisent des pratiques pédagogiques elles-mêmes différentes. Ainsi, l'évaluation est plus positive en Angleterre, par souci de préserver l'estime de soi de l'élève, et plus critique en France, où il s'agit de l'inciter à faire des efforts pour atteindre le niveau commun visé. De même, Maroussia Raveaud note qu'en maternelle, les exigences de maîtrise du corps sont formulées dans des situations inverses en France et en Angleterre. En France, le corps qui travaille doit être immobile, assis et silencieux, mais les enseignants montrent beaucoup plus de tolérance dans les moments de rassemblement. En Angleterre, les enfants peuvent écrire debout ou agenouillés sur leur chaise, mais le contrôle des corps est insistant lorsque les élèves se rassemblent auprès du professeur ou dans l'assembly du matin.

Les modèles scolaires français et anglais sont particulièrement importants car ils ont été exportés de par le monde, à travers la colonisation, et ils représentent deux pôles : un pôle universaliste, rationaliste et républicain, soucieux de la formation du citoyen critique ; un pôle individualiste, empiriste et communautaire, centré sur le développement global de l'enfant selon des valeurs communes.

Méritent également d'être soulignées les valeurs qui fondent les modèles asiatiques, notamment en Chine, en Corée du Sud et au Japon. Héritées de Confucius, considéré comme le " premier Maître ", "l'Éducateur ", elles prônent la valeur du savoir, l'apprentissage, l'étude et l'effort personnels, le développement moral et humain de soi-même (Jin et Cortazzi, RIE 50). Lorsque l'école de type occidental s'est implantée en Chine, elle a pu s'enraciner dans ces valeurs confucéennes avec lesquelles elle était compatible. 


\section{DEUX MOdÈles PERFORMANTS À CULTURES DIFFÉRENTES : la Finlande et la Corée du Sud}

L'existence de modèles scolaires à cohérence culturelle forte permet de comprendre pourquoi ces modèles sont divers et incite à penser qu'ils le resteront, malgré les pressions qu'exerce sur eux la globalisation. Ils ont traversé l'histoire et surmonteront les défis actuels. D’une certaine façon, les résultats de PISA peuvent conforter cette thèse. En effet, quand on échappe au tropisme finlandais, on s'aperçoit qu'un autre système scolaire, complètement différent, voire opposé, pourrait également servir de référence à qui pense l'école en termes de performances des élèves : le système sud-coréen. En 2003, la Finlande est première ou seconde dans toutes les matières évaluées par PISA et la Corée du Sud arrive toujours dans les quatre premiers. En 2006, la Finlande est première en mathématiques et sciences et n'est dépassée, en lecture, que par la Corée (quatrième en mathématiques, onzième en sciences). Seul Hong-Kong peut rivaliser avec la Corée du Sud comme dauphin de la Finlande en matière de performances des élèves. Or la Finlande et la Corée du Sud ont des modèles scolaires très différents.

En Finlande, le système scolaire est à la fois efficace et égalitaire durant les neuf années de l'école fondamentale (de 7 à 16 ans). L'école est publique ; il y a peu d'écoles privées et celles-ci, qui recrutent leurs élèves sur des critères essentiellement religieux ou linguistiques, sont financées par l'État et doivent respecter les critères nationaux. La très grande majorité des élèves fréquente l'école publique locale et il y a peu de différence de niveau entre les établissements. Les écoles disposent d'une grande autonomie et pratiquent une évaluation interne. Il n'existe pas d'inspection, ni de surveillants et l'administration scolaire est très légère. La concurrence est grande pour devenir professeur, bien que les salaires ne soient pas particulièrement élevés. Il n'existe aucune sélection dans l'école fondamentale et le plus souvent les élèves ne sont pas notés avant l'âge de douze ans ; l'évaluation est réellement formative et au service de l'apprentissage et on ne constate pas cette compétition permanente entre élèves qui caractérise beaucoup de systèmes, en particulier l'école française. Il n'y a pas de redoublement, sauf si les élèves veulent faire une dixième année pour améliorer leur formation avant d'aborder l'étape suivant de leur scolarité. La journée scolaire est courte et les devoirs à la maison sont légers. La clef de la réussite du modèle finlandais est sans doute la grande importance accordée aux élèves en difficulté, le souci pour le bien-être des élèves et l'absence de stress. L'école finlandaise s'attache à repérer très tôt la difficulté scolaire et à intervenir immédiatement, par une aide individualisée. En outre, l'autonomie de l'école lui permet de mettre en œuvre des dispositifs d'apprentissage très diversifiés; la seule obligation, intériorisée et acceptée par les enseignants, est que tous les élèves atteignent les objectifs. Malgré le lourd investissement dans la réussite de 
tous les élèves, les dépenses finlandaises en matière d'éducation sont inférieures à la moyenne de l'OCDE, ce qui est possible grâce à l'absence de redoublement et à la réduction drastique du personnel non-enseignant (Robert, 2008) ${ }^{4}$.

La structure du système sud-coréen est proche de celle du système finlandais : six années d'école primaire, trois années de middle school (et donc neuf années d'école fondamentale), puis trois années de high school. Mais, alors que le modèle finlandais est le symbole de l'école sans stress, le modèle sudcoréen rivalise avec le modèle japonais comme symbole de l'intense pression exercée sur les élèves. Le système est centralisé. À côté des écoles publiques existent des écoles privées, dont le coût est souvent prohibitif. Les enseignants sont évalués tous les deux ans. Les élèves sont très contrôlés, notamment en ce qui concerne uniforme et coupe de cheveux. L'enseignement est fondé sur la mémorisation, dès l'école maternelle. Il n'existe pas de redoublement, mais la concurrence pour entrer dans les «bonnes écoles» est intense : l'examen en fin d'école primaire a été remplacé par une loterie mais les élèves passent un examen en fin de middle school pour choisir leur high school et en fin de high school pour choisir leur université. Ces examens sont essentiels : la valeur d'une scolarité sur le marché du travail dépend en fait de la valeur de l'établissement fréquenté. Les élèves vont à l'école huit heures par jour, mais la plus grande partie d'entre eux fréquente ensuite des Hagwons, cours privés de soutien scolaire qu'ils suivent dès l'école élémentaire, et parfois dès l'école maternelle. Il n'est pas rare que des élèves de middle ou high school rentrent chez eux à minuit. Ce modèle sudcoréen a une efficacité incontestable et il a joué un rôle fondamental dans la réussite économique de la Corée du Sud. Mais il a un coût : pour les parents, très forte charge financière ; pour les enfants, compétition effrénée et précoce, conditions de vie difficiles et taux de suicide juvénile élevé.

Les modèles finlandais et sud-coréen reposent sur des valeurs radicalement différentes, liées à leur conditions géographique et à leur histoire : égalité et développement personnel en Finlande, concurrence et accumulation de connaissances en Corée du Sud. On peut se demander pourquoi deux modèles si opposés se révèlent également efficaces. Pour notre part, nous faisons l'hypothèse que c'est parce que le modèle ne crée pas, en soi, la performance de l'élève. Apprend qui s'engage dans une activité intellectuelle et la valeur du modèle scolaire dépend de sa capacité à induire, soutenir et nourrir cette activité (Charlot, 1999, 2005). Pour des raisons culturelles, la Finlande et la Corée du Sud y parviennent par des voies différentes, et de façon plus efficace que les États-Unis, plus riches mais où les élèves préfèrent la réussite sociale et sportive à la culture académique (Elliott, RIE 50).

Si l'on considère que les critères d'évaluation de PISA traduisent les exigences de la société globalisée en matière d'éducation et formation des jeunes, on aboutit à la conclusion que des modèles scolaires fondés sur des valeurs

4. Voir aussi Eurydice : http://eacea.ec.europa.eu/ressources/eurydice/eurybase/pdf/o_integral/FI_EN.pdf 
radicalement différentes peuvent répondre à ces exigences. Donc, la mondialisation ne mène pas, en tout cas pas nécessairement, à " une seule école ». Restent, toutefois, deux sources de doute.

\section{LES MODÈLES SCOLAIRES PEUVENT-ILS SE ROMPRE?}

Dans la mesure où les modèles scolaires ont été construits dans l'histoire, existe la possibilité qu'ils se déconstruisent en un autre moment de l'histoire. D'ailleurs, le modèle finlandais lui-même était élitiste jusqu'à sa réforme dans les années soixante-dix. Il n'est pas sûr que tous les modèles puissent, comme l'ont fait le finlandais et le sud-coréen, s'adapter aux impératifs de la globalisation en se régénérant de l'intérieur, c'est-à-dire en se transformant sans pour autant rompre avec la tradition culturelle qui les fonde. Ces impératifs, en effet, peuvent contredire directement les fondements du modèle. C'est le cas en Angleterre, où des programmes nationaux et des exigences cognitives ont été introduits dans un modèle reposant sur des valeurs communautaires et une éducation des comportements. C'est le cas également en France, où un principe de discrimination positive et des pressions en faveur de l'individualisation de l'enseignement bousculent fortement le modèle républicain, à la fois égalitaire et élitiste. Les chercheurs qui comparent l'éducation en France et en Angleterre tendent à insister sur la continuité des modèles. Ainsi, Osborn écrit : "L'étude conclut que les attitudes et traditions nationales, profondément ancrées, sont, dans une certaine mesure, réfractaires au changement. Malgré les réformes anglaises qui renforcent le pouvoir central et les tentatives françaises de créer plus de diversité, le système anglais continue à être caractérisé par l'individualité et la diversité, alors que le système français demeure une structure centralisée et bureaucratique»(RIE 50). Cependant, les chercheurs français et anglais ont surtout comparé les écoles élémentaires et maternelles des deux pays, alors que les plus fortes pressions s'exercent sur les établissements secondaires. En outre, cette conclusion n'est pas unanime. Elliott note que, en Russie, un rapport utilitaire à l'école, semblable à celui des jeunes occidentaux, tend à se substituer au rapport au savoir valorisé comme tel à l'époque soviétique, sous la forme " être une personne éduquée »(RIE 50). L'incertitude sur la résistance des modèles culturels historiquement construits demeure donc.

\section{QUAND UN MODÈLE SCOLAIRE EST EXPORTÉ DANS UN PAYS À CULTURE DIFFÉRENTE...}

La thèse de la cohérence culturelle des modèles scolaires se heurte à une seconde difficulté : en certaines zones du monde, les modèles scolaires occidentaux ont été implantés dans des pays où ils ne disposent pas d'une base 
culturelle solide. En Amérique du Nord ou en Australie, les immigrants ont emmené avec eux leur modèle d'école. En Asie, un modèle importé dans la deuxième moitié du XIX ${ }^{\mathrm{e}}$ siècle a trouvé dans le confucianisme les bases culturelles lui permettant de prospérer. Mais qu'en est-il en Amérique du Sud et en Afrique ?

Dans certains pays d'Amérique du Sud, à forte prédominance européenne, la situation a été semblable à celle de l'Amérique du Nord : le modèle occidental d'école s'est enraciné sans difficulté. C'est le cas, par exemple, en Uruguay et en Argentine, où l'école primaire obligatoire et gratuite est née à la même époque qu'en France, voire un peu avant. Mais la situation est très différente dans les pays où une grande partie de la population est d'origine indienne ou est issue de l'esclavage des Noirs. Ces populations ont des traditions orales, alors que l'école occidentale importée repose sur l'écrit, et leurs communautés, lieux de résistance au blanc, ont des raisons de se méfier de son école. En outre, dans ces pays, la question scolaire est liée directement à l'énorme inégalité qui structure la société, de sorte que leur problème est à la fois de scolariser tous les jeunes, de construire " une seule école » au niveau national et de s’insérer dans la globalisation.

Le cas du Brésil est particulièrement intéressant, dans la mesure où c'est à la fois un pays riche déjà engagé dans la concurrence mondiale, un pays culturellement métissé et l'un des pays les plus inégalitaires du monde. Un tel pays doit construire sur un temps court un système scolaire et universitaire que des pays comme la France ou l'Angleterre ont pu construire au long d'un siècle et demi. Aujourd'hui, il scolarise $97 \%$ des jeunes dans l'enseignement fondamental (neuf années), environ $40 \%$ dans l'enseignement médio en trois ans (de type lycée) et il dispose d'un système universitaire de niveau correct. Mais il y a, en fait, deux écoles au Brésil : l'école privée à but lucratif pour les classes moyennes et une école publique, mal soutenue par les autorités locales et nationales, pour le peuple. Au niveau supérieur, les universités privées ont plus d'étudiants que les universités publiques. Mais la répartition des flux universitaires est paradoxale : dans la mesure où on entre à l'université par un concours, très sélectif dans le cas des universités fédérales, les élèves issus des écoles privées, dont le niveau moyen est meilleur, font des études supérieures gratuites dans les universités fédérales et les élèves issus des écoles publiques payent leurs études dans des universités privées - et, pour ce faire, travaillent en même temps qu'ils étudient. On peut dire que, au Brésil, il existe à la fois deux écoles et une école. Il y a deux écoles : publique et privée. Mais les écoles privées et les universités fédérales cherchent à fonctionner selon les normes de l'école de la société globalisée.

L'exemple du Brésil met en évidence une nouvelle donnée du problème, qui apparaît mal quand on se place dans la seule perspective culturelle : la question «Un seul monde, une seule école? » ne doit pas être traitée seulement dans l'opposition modèle scolaire national versus modèle globalisé, elle doit aussi prendre en compte les processus de différenciation interne des modèles nationaux, sous l'effet de la globalisation. Une analyse des modèles scolaires 
africains mènerait sans doute à la même conclusion. Les pays africains doivent construire, dans des conditions financières et démographiques très difficiles, une école pour tous et celle-ci est loin de pouvoir rivaliser avec les écoles occidentales ou asiatiques. Mais, en même temps, y apparaît, pour les enfants des classes les plus favorisées, une école privée qui tente d'adopter les standards globaux. Ainsi, le Kenya est « en marche vers la scolarisation universelle ", grâce à l'instauration de la gratuité de l'école primaire en 2003 et de l'enseignement secondaire en 2008, et au prix d'un énorme effort financier (plus de $25 \%$ du budget national consacré à l'éducation). Mais, parallèlement, " les écoles privées permettent aux plus riches de garantir à leurs enfants une école de qualité », et on assiste à un « succès sans précédent des universités privées » (Charton, RIE 50). L'Inde, dont $20 \%$ des établissements scolaires sont des écoles privées sans subvention, semble engagée dans la même évolution.

\section{UN NOUVEL ÉLÉMENT : LA DIFFÉRENCIATION INTERNE DES SYSTÈMES SCOLAIRES}

La globalisation tend ainsi à produire un double mouvement. D'une part, elle induit des convergences entre modèles scolaires nationaux (nettes, par exemple, entre la France et l'Angleterre). D’autre part, elle produit des différenciations scolaires internes à ces pays.

Certaines différences sont antérieures à la globalisation : entre villes et campagnes, entre filles et garçons. La globalisation tend à accroître les premières, qui dépendent de l'offre scolaire, et à diminuer les secondes, effets de la demande. D'autres différences sont liées à des caractéristiques des populations scolarisées, en termes d'origine, de mode de vie, d'âge : enfants de migrants, enfants des rues, enfants des communautés nomades, adultes, etc. L'exigence d'élévation du niveau de formation de toute la population, qui fait partie du programme de la globalisation, amène à s'occuper de ces populations, mais elles sont souvent prises en charge par des dispositifs spécifiques.

Mais la globalisation a surtout pour effet de produire ou d'aggraver des différenciations structurelles liées à l'inégalité sociale. Il en existe trois grands modèles. Premièrement, le modèle brésilien : éclatement du système en deux réseaux, public et privé, scolarisant des élèves socialement différents. Deuxièmement, le modèle japonais / sud-coréen : effet déterminant de l'école après l'école (comme les Hagwons), articulé sur une hiérarchisation des établissements. Troisièmement, le modèle français : à l'intérieur même de l'école publique, différenciation de fait de la qualité des établissements et répartition masquée d'élèves socialement différents dans des classes distinctes. Combinées à ces différenciations sociales, s'ajoutent des différenciations géographiques : entre le Nord et le Sud de l'Espagne, du Brésil, de l'Allemagne ; entre l'Ouest et l'Est de l'Allemagne ou de la Chine. 
Ces effets de différenciation nous rappellent que la globalisation n'est pas une mondialisation. Sa logique est celle des réseaux et des flux et non celle de l'unification solidaire d'un territoire mondial. Dès lors, on ne saurait s'étonner que tende à se construire, traversant les cultures nationales, un réseau d'éducation défini par des standards globaux : "une seule école ». C’est ce réseau qui se développe dans de nombreux pays, sous forme d'écoles privées à but lucratif, de cours de langue anglaise, d'écoles publiques élitistes, et qui utilise des structures de tutorat multinationales (Kumon, Sylan, Acadomia) ou le tutorat par Internet (par exemple, des tuteurs en Inde vendent leurs services à des enfants américains $)^{5}$. Mais, parallèlement, la globalisation tend à faire éclater les modèles nationaux en deux écoles : celle de la globalisation et l'autre. Les cultures nationales survivent, comme vestiges et nuances dans l'école globalisée, comme problèmes ou résistances dans l'école en voie de globalisation, comme points d'appui de l'école des plus pauvres.

Le modèle finlandais, tout à la fois culturellement enraciné, démocratique et efficace constitue une exception heureuse qui prouve que modernité et performance n'impliquent pas nécessairement concurrence, élitisme et dégradation des conditions de vie des enfants. Sans doute est-ce pour cela qu'il fait rêver : rêve d'une école où "qualité » serait synonyme de sens et plaisir d'apprendre et où «mondialisation » signifierait solidarité entre les êtres humains.

\section{BIBLIOGRAPHIE}

CHARLOT B. (1999) : Du Rapport au savoir. Éléments pour une théorie, Paris: Anthropos.

CHARLOT B. (2005) : Relação com o saber, Formação dos professores e Globalização : questões para a educação hoje, Porto Alegre : ARTMED.

CHARLOT B. (2007) : "Educação e Globalização. Uma tentativa de colocar ordem no debate ", Sísifo. Revista de Ciências da Educação da Universidade de Lisboa, no 4, sept.-déc, p. 129-136. Consultable en ligne : http://sisifo.fpce.ul.pt/?r=13\&p=129

CIEP (2009) : Colloque international Un seul monde, une seule école? Les modèles scolaires à l'épreuve de la mondialisation, contributions consultables en ligne : http:// www.ciep.fr/ries/colloque-2009/index.php.

RAVEAUD M. (2006) : De l'enfant au citoyen. La construction de la citoyenneté à l'école en France et en Angleterre, Paris : PUF.

Revue internationale d'éducation de Sèvres (2009) : " En classe : pratiques pédagogiques et valeurs culturelles ", no 50, avril. Articles de : Alexander, Elliott, Jin et Cortazzi, Longo et Roche, MacNess, Osborn, Planel, Charton.

ROBERT P. (2008) : La Finlande : un modèle éducatif pour la France? Les secrets d'une réussite, Issy-les-Moulineaux : ESF.

5. Voir, sur le site du CIEP du colloque, le texte de Mark Bray : http://www.ciep.fr/ries/colloque-2009/index.php/. 
